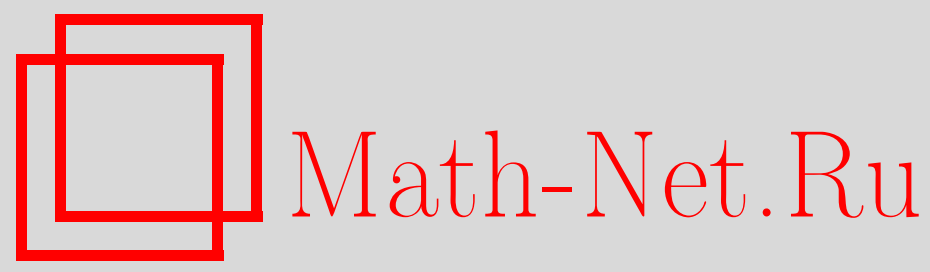

Б. Д. Гельман, Непрерывные аппроксимации многозначных отображений и неподвижные точки, Матем. заметки, 2005, том 78, выпуск 2, 212-222

DOI: https://doi.org/10.4213/mzm2649

Использование Общероссийского математического портала Math-Net.Ru подразумевает, что вы прочитали и согласны с пользовательским соглашением http://www.mathnet.ru/rus/agreement

Параметры загрузки:

IP : 52.87 .193 .239

26 апреля 2023 г., 15:04:49

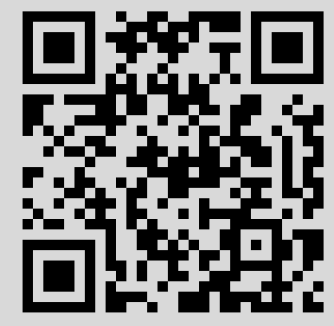




\title{
НЕПРЕРЫВНЫЕ АППРОКСИМАЦИИ МНОГОЗНАЧНЫХ ОТОБРАЖЕНИЙ И НЕПОДВИЖНЫЕ ТОЧКИ
}

\author{
Б. Д. Гельман
}

\begin{abstract}
Настоящая статья посвящена доказательству теоремы о неподвижной точке для вполне непрерьвных многозначных отображений, определенных на ограниченном выпуклом замкнутом подмножестве $X$ гильбертова пространства $H$ и удовлетворяющих тангенциальному условию $F(x) \cap\left(x+T_{X}(x)\right) \neq \varnothing$, где $T_{X}(x)$ - касательньй конус к множеству $X$ в точке $x$. Доказательство этой теоремы основывается на методе однозначных аппроксимаций многозначных отображений. В статье рассматривается простой подход к построению однозначных аппроксимаций многозначных отображений. Этот подход позволяет не только упростить доказательства известных ранее теорем, но и получить новые утверждения, которые необходимы для доказательства основной теоремы статьи.
\end{abstract}

Библиография: 12 названий.

Настоящая статья посвящена доказательству теоремы о неподвижной точке для вполне непрерывных многозначных отображений, определенных на ограниченном выпуклом замкнутом подмножестве $X$ гильбертова пространства $H$ и удовлетворяюших тангенциальному условию

$$
F(x) \cap\left(x+T_{X}(x)\right) \neq \varnothing \quad \text { для любого } x \in X,
$$

где $T_{X}(x)$ - касательньй конус к множеству $X$ в точке $x$.

Такого типа теоремы известны в случае, когда $X$ является выпукльм компактом (см., например, [1]) или компактом более сложной топологической структуры (см., например, [2]). Их доказательства существенно опираются на лемму Ки Фаня и носят аналитический характер. Эти теоремы находят приложения в математической экономике и теории дифференциальных включений (см. [3]-[5]).

Доказательство основной теоремы статьи основывается на методе однозначных аппроксимаций и сечений многозначных отображений. Хорошо известна важная роль, которую играют однозначные аппроксимации и сечения в теории неподвижных точек многозначных отображений (см., например, [6]-[9] и др.). В пп. 1-3 данной статьи рассматривается простой подход к построению однозначных аппроксимаций. Этот подход позволяет не только упростить доказательства известных ранее теорем, но и получить новые утверждения, которые необходимы для доказательства основной теоремы статьи.

Работа выполнена при поддержке Российского фонда фундаментальных исследований, грант № 02-01-00189. 
1. Основные факты теории многозначных отображений. Пусть $Y$ - подмножество нормированного пространства $E$, обозначим

$\mathrm{P}(Y)$ - множество всех непустых подмножеств в $Y$;

$\mathrm{V}(Y)$ - множество всех непустых вьпуклых подмножеств в $Y$;

$\mathrm{Cv}(Y)$ - множество всех непустых замкнутых выпуклых подмножеств в $Y$;

$\mathrm{Kv}(Y)$ - множество всех непустых компактных выпуклых подмножеств в $Y$.

Многозначное отображсение метрического пространства $X$ в метрическое пространство $Y$ - это соответствие, сопоставляющее каждой точке $x \in X$ непустое подмножество $F(x) \subset Y$, называемое образом точки $x$. В дальнейшем, если образы многозначного отображения $F$ являются вьпуклыми, то будем записывать это следующим образом, $F: X \rightarrow \mathrm{V}(Y)$. Аналогично, обозначение $F: X \rightarrow \mathrm{Cv}(Y)$ (соответственно $F: X \rightarrow \mathrm{Kv}(Y))$ означает, что образы $F(x)$ являются выпуклыми замкнутыми (соответственно компактными) множествами.

Графиком многозначного отображения $F: X \rightarrow \mathrm{P}(Y)$ называется множество

$$
\Gamma_{X}(F)=\{(x, z) \mid x \in X, \quad z \in F(x)\} \subset X \times Y
$$

Всюду в дальнейшем многозначные отображения обозначаются прописными, а однозначные - строчньги буквами.

ОПРЕДЕЛЕНИЕ 1. Многозначноеотображение $F: X \rightarrow \mathrm{P}(Y)$ назьвается полунепрерывным снизу в точке $x_{0} \in X$, если для любого открытого множества $V \subset Y$ такого, что $F\left(x_{0}\right) \cap V \neq \varnothing$, найдется открытая окрестность $U$ точки $x_{0}$ такая, что $F(x) \cap V \neq \varnothing$ для любого $x \in U$. Если $F$ полунепрерьвно снизу в каждой точке $x \in X$, то оно называется полунепрерывным снизу.

ПРЕДЛОЖЕНИЕ 1. Следующие условия әквивалентны:

1) F полунепрерывно снизу;

2) для любого открытого множсества $V \subset Y$ полный прообраз этого множества

$$
F_{\Pi}^{-1}(V)=\{x \in X \mid F(x) \cap V \neq \varnothing\}
$$

является открытым множсеством в $X$;

3) для любого замкнутого мноэсества $W \subset Y$ малый прообраз әтого множсества

$$
F_{\mathrm{M}}^{-1}(W)=\{x \in X \mid F(x) \subset W\}
$$

является замкнутым мнохеством в $X$.

ДокАЗАТЕЛЬСтво этого утверждения содержится, например, в [10].

Приведем еще один, по-видимому новьй, критерий полунепрерывности снизу многозначного отображения.

Пусть $F: X \rightarrow \mathrm{P}(Y)$ - многозначное отображение, $\Gamma_{X}(F)$ - график этого отображения. Естественно определены проекции

$$
t: \Gamma_{X}(F) \rightarrow X, \quad t(x, y)=x, \quad r: \Gamma_{X}(F) \rightarrow Y, \quad r(x, y)=y
$$

Очевидно, что для любого $x \in X$ справедливо равенство: $F(x)=r\left(t^{-1}(x)\right)$. 
ПРЕДЛОЖЕНИЕ 2. Многозначное отображсене $F$ полунепрерывно снизу тогда и только тогда, когда проекиия $t$ является открытым отображсением.

ДокАЗАТЕЛЬСтво. Необходимость. Пусть множество $W \subset \Gamma_{X}(F)$ - открытое множество и $t(W)=U$. Покажем, что множество $U$ открыто в $X$. Предположим противное, тогда существует точка $x_{0} \in U$ такая, что $\left(x_{0}, y_{0}\right) \in W$, и для любого $\delta>0$ найдется точка $x_{\delta}, \rho\left(x_{\delta}, x_{0}\right)<\delta$, причем $\left(x_{\delta} \times F\left(x_{\delta}\right)\right) \cap W=\varnothing$.

$\mathrm{C}$ другой стороны, так как множество $W$ открыто, то существуют такие числа $\delta_{0}>0$, $\eta_{0}>0$, что произведение окрестностей $U_{\delta_{0}}\left(x_{0}\right) \times U_{\eta_{0}}\left(y_{0}\right) \subset W$. Из полунепрерьвности снизу многозначного отображения $F$ вытекает, что найдется такое $\delta_{1}>0$, что для любого $x \in U_{\delta_{1}}\left(x_{0}\right)$ пересечение $F(x) \cap U_{\eta_{0}}\left(y_{0}\right) \neq \varnothing$. Следовательно, для достаточно малых $\delta$ пересечение $\left(x_{\delta} \times F\left(x_{\delta}\right)\right) \cap W \neq \varnothing$. Полученное противоречие и доказывает необходимость.

Достаточность. Пусть $t$ является открытым отображением. Рассмотрим открытое множество $V \subset Y$ и докажем открытость множества

$$
F_{\Pi}^{-1}(V)=\{x \in X \mid F(x) \cap V \neq \varnothing\} .
$$

Пусть точка $x_{0} \in F_{\Pi}^{-1}(V)$, тогда найдется точка $y_{0}$ такая, что $y_{0} \in\left(F\left(x_{0}\right) \cap V\right)$. В силу открытости множества $V$ существует число $\eta>0$ такое, что $U_{\eta}\left(y_{0}\right) \subset V$. Рассмотрим множество

$$
W=\left(U_{\varepsilon}\left(x_{0}\right) \times U_{\eta}\left(y_{0}\right)\right) \cap \Gamma_{X}(F) .
$$

Очевидно, что это множество открыто в $\Gamma_{X}(F)$. Тогда множество $U=t(W)$ также является открытьм и $x_{0} \in U$. Пусть $x \in U$, тогда существует точка $y \in F(x)$ такая, что $(x, y) \in W$, т.е. $x \in F_{\Pi}^{-1}(V)$. Следовательно, точка $x_{0}$ лежит в $F_{\Pi}^{-1}(V)$ вместе со своей открытой окрестностью $U$, что и доказывает утверждение.

СлЕДСТВИЕ 1. Если график многозначного отображения $F$ открытое множество в $X \times Y$, то отобрахсение $F$ является полунепрерывным снизу.

ДокАЗАТЕЛЬСТВО вытекает из открытости проекции $t$.

Рассмотрим другой класс многозначных отображений.

ОПРЕДЕЛЕНИЕ 2. Многозначное отображение $F: X \rightarrow \mathrm{P}(Y)$ называется полунепрерывным сверху в точке $x_{0} \in X$, если для любого открытого множества $V \subset Y$, $V \supset F\left(x_{0}\right)$, существует открытая окрестность $U$ точки $x_{0}$ такая, что $F(U) \subset V$. Если $F$ полунепрерывно сверху в каждой точке $x \in X$, то оно назьвается полунепрерывным сверху.

ПРЕДЛОЖЕНИЕ 3. Следующие условия әквивалентны:

1) F полунепрерывно сверху;

2) для любого открытого мнохсества $V \subset Y$ малый прообраз әтого множсества

$$
F_{\mathrm{M}}^{-1}(V)=\{x \in X \mid F(x) \subset V\}
$$

является открытым множеством в $X$;

3) для любого замкнутого множества $W \subset Y$ полный прообраз этого множества

$$
F_{\Pi}^{-1}(W)=\{x \in X \mid F(x) \cap W \neq \varnothing\}
$$

является замкнутым мнохеством в $X$. 
ДокАЗАТЕЛьСТво этого утверждения содержится, например, в [10].

ОПРЕДЕЛЕНИЕ 3. Многозначное отображение $F: X \rightarrow \mathrm{P}(Y)$ назьвается непрерывным, если оно одновременно является полунепрерывным и сверху и снизу.

Очевидно, что свойства непрерывных многозначных отображений вытекают из соответствующих свойств полунепрерывных сверху и снизу отображений.

Рассмотрим класс многозначных отображений, важньй для дальнейших рассмотрений.

Пусть $X$ - метрическое пространство, $Y$ - нормированное пространство, $F: X \rightarrow$ $\mathrm{P}(Y)$ - некоторое многозначное отображение.

ОПРЕДЕЛЕНИЕ 4 . Будем говорить, что $F$ является $\mathscr{U}$ - отображением, если оно удовлетворяет двум условиям:

a) график $\Gamma_{X}(F)$ является открытым множеством в $X \times Y$;

b) для любой точки $x \in X$ образ $F(x)$ является выпуклым множеством.

Обозначим множество всех $\mathscr{U}$-отображений из $X$ в $Y$ символом $\mathscr{U}(X, Y)$. Многозначные отображения, принадлежашие $\mathcal{U}(X, Y)$, обладают следуюшими свойствами.

Свойство 1. Если $F \in \mathscr{U}(X, Y)$, то оно является полунепрерывным снизу.

ДоКАЗАТЕЛЬСТВО этого свойства вытекает из следствия 1.

Пусть $A$ - подмножество в $Y$. Обозначим через $U_{\varepsilon}(A) \varepsilon$-окрестность множества $A$, т.e.

$$
U_{\varepsilon}(A)=\{y \in Y \mid \rho(y, A)<\varepsilon\} .
$$

Свойство 2. Если $F: X \rightarrow \mathrm{V}(Y)$ - полунепрерывное снизу многозначное отображсение, то для любого $\varepsilon>0$ многозначное отображение $F^{\varepsilon}: X \rightarrow \mathrm{V}(Y)$, $F^{\varepsilon}(x)=U_{\varepsilon}(F(x))$, является $\mathscr{U}$-отображсением.

ДОКАЗАТЕЛЬСТВО очевидно.

Свойство 3. Пусть многозначные отображения $F_{1}, \ldots, F_{n} \in \mathscr{U}(X, Y)$. Если для любой точки $x \in X$ пересечение $\bigcap_{i=1}^{n} F_{i}(x) \neq \varnothing$, то многозначное отобрахсение $\bigcap_{i=1}^{n} F_{i} \in \mathscr{U}(X, Y)$.

ДоКАЗАТЕЛЬСТВО очевидно.

2. Непрерывные сечения и аппроксимации многозначных отображений. Пусть $X$-метрическоепространство, $Y$-нормированноепространство, $F: X \rightarrow \mathrm{P}(Y)$ некоторое многозначное отображение.

ОПРЕДЕЛЕНИЕ 5. Непрерьвное отображение $f: X \rightarrow Y$ назьвается сечением многозначного отображения $F$, если для любой точки $x \in X$ выполнено включение $f(x) \in F(x)$.

Приведем формулировку классической теоремы Майкла о сечении [11].

Теорема 1. Пусть $E$ - банахово пространство, $F: X \rightarrow \mathrm{Cv}(E)$ - полунепрерывное снизу многозначное отображсние, $A$ - замкнутое подмножество в $X$. Eсли $f: A \rightarrow E$ непрерывное сечение $\left.F\right|_{A}$, то у $F$ существует непрерывное сечение $g: X \rightarrow E$ такое, что $\left.g\right|_{A}=f$. 
ОПРедЕЛЕниЕ 6. Многозначное отображение $G: X \rightarrow \mathrm{P}(Y)$ назьвается $\varepsilon$-annрокcuмачией многозначного отображения $F$, если график $\Gamma_{X}(G)$ отображения $G$ принадлежит $\varepsilon$-окрестности графика $\Gamma_{X}(F)$ многозначного отображения $F$.

В случае, если $G$ является однозначным непрерьвным отображением, говорят, что $G$ - однозначная $\varepsilon$-апшроксимация многозначного отображения $F$.

Теорема 2. Пусть $F: X \rightarrow \mathrm{Cv}(Y)$ - полунепрерывное сверху многозначное отображение. Тогда для любого $\varepsilon>0$ существует полунепрерывное снизу многозначное отображсние $F_{\varepsilon}: X \rightarrow \mathrm{Cv}(Y)$, удовлетворяющее следующим условиям:

1) $F_{\varepsilon}(x)=\overline{\sum_{j \in J} \varphi_{j}(x) A_{j}}$, əде $\left\{\varphi_{j}\right\}_{j \in J}$ - разбиение единицы, построенное по некоторому открытому локально конечному покрытию пространства $X$; $\left\{A_{j}\right\}_{j \in J},-$ выпуклые замкнутые множества в $Y$;

2) $F(x) \subset F_{\varepsilon}(x)$ для любого $x \in X$;

3) график $\Gamma_{X}\left(F_{\varepsilon}\right) \subset U_{\varepsilon}\left(\Gamma_{X}(F)\right)$;

4) $F_{\varepsilon}(X) \subset \overline{\operatorname{co}}(F(X))$.

ДокАЗАТЕльСТво. Пусть $\varepsilon$ - произвольное положительное число. В силу полунепрерьвности сверху многозначного отображения $F$, для любой точки $x \in X$ существует положительное число $\delta(x)$ такое, что для любой точки $x^{\prime} \in U_{\delta(x)}(x)$ выполнено включение $F\left(x^{\prime}\right) \subset U_{\varepsilon / 2}(F(x))$. Без ограничения общности будем считать, что $0<\delta(x)<\varepsilon$. Пусть $\eta(x)=\delta(x) / 4$. Рассмотрим открытое покрытие $\tau=\left\{U_{\eta(x)}(x)\right\}_{x \in X}$. Выберем из него локально конечное подпокрытие

$$
\sigma=\left\{V_{j}=U_{\eta\left(x_{j}\right)}\left(x_{j}\right)\right\}_{j \in J}
$$

Пусть функции $\left\{\varphi_{j}\right\}_{j \in J}$ образуют непрерьвное разбиение единицы, подчиненное этому покрытию. Рассмотрим многозначное отображение $F_{\sigma}$, где

$$
F_{\sigma}(x)=\sum_{j \in J} \varphi_{j}(x) \overline{\operatorname{co}} F\left(V_{j}\right)
$$

Очевидно, что это отображение полунепрерывно снизу.

Проверим для отображения $F_{\sigma}$ выполнение условия 2$)$. Пусть $x$-произвольная точка из $X$. Значение $\varphi_{j}(x) \neq 0$ тогда и только тогда, когда $x \in V_{j}$. Пусть $j_{1}, j_{2}, \ldots, j_{s}-$ индексы всех функций, для которых $\varphi_{j}(x) \neq 0$. Тогда

$$
F_{\sigma}(x)=\sum_{i=1}^{s} \varphi_{j_{i}}(x) \overline{\operatorname{co}} F\left(V_{j_{i}}\right), \quad \text { где } \quad x \in \bigcap_{i=1}^{s} V_{j_{i}} .
$$

Следовательно, $F(x) \subset F\left(V_{j_{i}}\right) \subset \overline{\mathrm{co}} F\left(V_{j_{i}}\right)$. Тогда $F(x) \subset F_{\sigma}(x)$.

Покажем, что отображение $F_{\sigma}$ удовлетворяет условию 3$)$. Пусть $x \in V_{j_{i}} \subset X$, $i=1,2, \ldots, s$. Множество $V_{j_{i}}=U_{\eta_{x_{i}}}\left(x_{i}\right)$, где $i=1,2, \ldots, s$. Пусть $\eta_{x_{k}}=\max _{1 \leqslant i \leqslant s} \eta_{x_{i}}$, тогда $x \in U_{\eta_{x_{k}}}\left(x_{i}\right)$ для любого $i=1,2, \ldots, s$. Тогда $x_{i} \in U_{2 \eta_{k}}\left(x_{k}\right)$ для любого

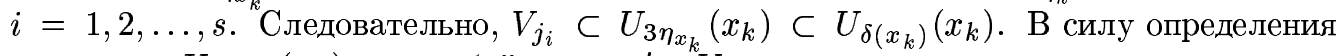
множества $U_{\delta\left(x_{k}\right)}\left(x_{k}\right)$ для любой точки $x^{\prime} \in V_{j_{i}}$ вьполнено включение

$$
F\left(x^{\prime}\right) \subset U_{\varepsilon / 2}\left(F\left(x_{k}\right)\right)
$$


Так как множество $F\left(x_{k}\right)$ выпукло, то

$$
F_{\sigma}(x)=\sum_{i=1}^{s} \varphi_{j_{i}}(x) \overline{\operatorname{co}} F\left(V_{j_{i}}\right) \subset \overline{U_{\varepsilon / 2}\left(F\left(x_{k}\right)\right)} .
$$

Рассмотрим отображение $F_{\varepsilon}(x)=\overline{F_{\sigma}(x)}$. Очевидно, что это отображение удовлетворяет условиям 1) и 2) теоремы. Условие 3 ) вытекает из того, что

$$
\overline{F_{\sigma}(x)} \subset \overline{U_{\varepsilon / 2}\left(F\left(x_{k}\right)\right)} \subset U_{\varepsilon}\left(F\left(x_{k}\right)\right) .
$$

Так как $\rho\left(x, x_{k}\right)<\eta_{s}<\varepsilon$, то график $\Gamma\left(F_{\varepsilon}\right) \subset U_{\varepsilon}(\Gamma(F))$.

Условие 4) вытекает из того, что

$$
F_{\sigma}(x) \subset \operatorname{co}\left\{\overline{\operatorname{co}} F\left(V_{j_{1}}\right), \ldots, \overline{\operatorname{co}} F\left(V_{j_{s}}\right)\right\} \subset \overline{\operatorname{co}} F(X)
$$

Теорема доказана.

СледСТВИЕ 2. Пусть $E$ - банахово пространство и $A$ - замкнутое подмножсество $X$. Если многозначное отображсение $F: X \rightarrow \mathrm{Cv}(E)$ полунепрерывно сверху и отображение $f: A \rightarrow E$ является непрерывным сечением многозначного отображсения $\left.F\right|_{A}$, то для любого $\varepsilon>0$ существует $\varepsilon$-аппроксимация $f_{\varepsilon}$ такая, что $f_{\varepsilon}(x)=f(x)$ для любой точки $x \in A u f_{\varepsilon}(X) \subset \overline{\mathrm{co}}(F(X))$.

ДокАЗАТЕльСтво. Пусть $F_{\varepsilon}$ - многозначная полунепрерывная снизу $\varepsilon$-аппроксимация, существующая в силу теоремы 2. Тогда у него существует непрерывное однозначное сечение, которое является непрерывным продолжением $f$. Очевидно, что это сечение и будет искомым отображением $f_{\varepsilon}$.

3. Однозначные сечения и аппроксимации пересечения многозначных отображений. Пусть $X$ - метрическое пространство, $E$ - банахово пространство, $F: X \rightarrow \mathrm{Cv}(E)$ - полунепрерывное снизу многозначное отображение, $G: X \rightarrow \mathrm{V}(E)-$ $\mathcal{U}$-отображение. Пусть для любого $x \in X$ пересечение $F(x) \cap G(x) \neq \varnothing$. Обозначим $F \cap G$ многозначное отображение, определенное условием $(F \cap G)(x)=F(x) \cap G(x)$.

Теорема 3. Пусть $A$ - замкнутое подмножсество в $X$. Если $f: A \rightarrow E-$ прерывное сечение $\left.(F \cap G)\right|_{A}$, то у многозначного отобрахсения $F \cap G$ существует непрерывное сечение $g: X \rightarrow E$ такое, что $\left.g\right|_{A}=f$.

ДокАЗАТЕЛЬСТво. Пусть $f_{1}$ - произвольное непрерьвное сечение многозначного отображения $F$, которое является непрерывным продолжением отображения $f$ на все пространство $X$. Такое сечение существует в силу теоремы 1 . Тогда, в силу открытости графика отображения $G$, существует открытое множество $U \supset A$ такое, что $\left.f_{1}\right|_{U}$ является сечением многозначного отображения $\left.G\right|_{U}$.

Пусть точка $x \in B=X \backslash U$, выберем произвольно точку $y_{x} \in(F(x) \cap G(x))$. Рассмотрим произвольное сечение $f_{x}$ многозначного отображения $F$, которое удовлетворяет условию: $f_{x}(x)=y_{x}$. В силу открытости графика отображения $G$, существует открытая окрестность $U(x)$ точки $x$ такая, что $f_{x}\left(x^{\prime}\right) \in G\left(x^{\prime}\right)$ для любой точки $x^{\prime} \in U(x)$ и $U(x) \cap A=\varnothing$. Очевидно, что такое отображение $f_{x}$ может быть построено для любой точки $x \in B$. Тогда семейство $\{U(x)\}_{x \in B}$ образует открытоепокрытиемножества $B$. Выберем из этого покрытия локально конечное подпокрытие $\left\{U\left(x_{\alpha}\right)\right\}_{\alpha \in J}$. 
Тогда семейство $\left\{U\left\{U\left(x_{\alpha}\right)\right\}_{\alpha \in J}\right\}$ образует локально конечное покрытие пространства $X$. Пусть функции $\left\{\varphi(x) ;\left\{\varphi_{\alpha}\right\}_{\alpha \in J}\right\}$ образуют разбиение единицы, построенное по этому покрытию. Рассмотрим отображение $g: X \rightarrow E$, определенное по правилу

$$
g(x)=\varphi(x) f_{1}(x)+\sum_{\alpha \in J} \varphi_{\alpha}(x) f_{x_{\alpha}}(x) .
$$

Нетрудно доказать, что в силу выпуклости образов многозначных отображений $F$ и $G$, построенное отображение $g$ будет искомьм сечением. Теорема доказана.

Теорема 4. Пусть $F_{i}: X \rightarrow \operatorname{Cv}(E), i=0,1, \ldots, k,-$ полунепрерывные снизу многозначные отображсения, $G_{j}: X \rightarrow \mathrm{Cv}(E), j=1, \ldots, s,-$ полунепрерывные сверху многозначные отображсения. Если для любого $x \in X$ пересечение

$$
\left(\bigcap_{i=0}^{k} F_{i}(x)\right) \cap\left(\bigcap_{j=1}^{s} G_{j}(x)\right) \neq \varnothing,
$$

то для любого $\varepsilon>0$ существует непрерывное отображсение $f_{\varepsilon}: X \rightarrow$ Е удовлетворяющее следующим условиям:

1) $f_{\varepsilon}$ является непрерывным сечением многозначного отображсения $F_{0}$;

2) для любого $x \in X$ расстояние $\rho\left(f_{\varepsilon}(x), F_{i}(x)\right)<\varepsilon, \quad i=1, \ldots, k$;

3) отображение $f_{\varepsilon}$ является $\varepsilon$-аппроксимацией многозначных отображсений $G_{j}, j=1, \ldots, s$.

ДокАЗАТЕльСтво. В силу теоремы 2 существуют полунепрерьвные снизу многозначные отображения $\widetilde{G}_{j}$ такие, что $G_{j}(x) \subset \widetilde{G}_{j}(x)$ для любого $x \in X$ и график $\Gamma_{X}\left(\widetilde{G}_{j}\right)$ $\subset U_{\varepsilon / 2}\left(\Gamma_{X}\left(G_{j}\right)\right)$. Тогда многозначные отображения $\widetilde{G}_{j}^{\varepsilon / 2}$, где $\widetilde{G}_{j}^{\varepsilon / 2}(x)=U_{\varepsilon / 2}\left(\widetilde{G}_{j}(x)\right)$, будут $\mathscr{U}$-отображениями.

Аналогично, $\mathscr{U}$-отображениями будут многозначные отображения $F_{i}^{\varepsilon}$ при $i=1, \ldots, k$. Рассмотрим пересечение

$$
\left(\bigcap_{i=1}^{k} F_{i}^{\varepsilon}(x)\right) \cap\left(\bigcap_{j=1}^{s} \widetilde{G}_{j}^{\varepsilon / 2}(x)\right)=G(x) .
$$

Очевидно, что многозначное отображение $G$ также является $\mathscr{U}$-отображением и $G(x) \cap$ $F_{0}(x) \neq \varnothing$. В силу теоремы 3 многозначное отображение $G \cap F_{0}$ имеет непрерывное сечение, которое и будет искомым.

4. Об одной теореме о неподвижной точке. Пусть $Y$ - нормированное пространство, $X$ - подмножество в $Y$.

Многозначное отображение $F: X \rightarrow K(Y)$ назьвается вполне непрерывным, если

1) $F$ полунепрерьвно сверху;

2) множество $\overline{F(B)}$ является компактньм в $Y$ для любого ограниченного множества $B \subset X$.

Точка $x_{0} \in X$ назьвается неподвижной точкой многозначного отображения $F$, если $x_{0} \in F\left(x_{0}\right)$.

Нам понадобятся следующие леммы.

Пусть $E$ - банахово пространство, $X$ - ограниченное замкнутое подмножество в $E$. 
Лемма 1. Пусть $F: X \rightarrow P(E)$ - многозначное отображение, удовлетворяющее следуюшим условиям:

1) существует открытое мнохсество $U$, содержсащее $X$ и многозначное отображсение $\widetilde{F}: U \rightarrow P(E),\left.\widetilde{F}\right|_{X}=F$, такие, что $x \notin \widetilde{F}(x)$ для любой точки $x \in(U \backslash X)$

2) существует точка $x_{0} \in X$ такая, что $x_{0} \in \tilde{F}(x)$ для любой точки $x \in U$;

3) для любой точки $x \in U$ мнохество $\widetilde{F}(x)$ стягиваемо по лучам к точке $x_{0}$. Пусть $f: X \rightarrow E$ - вполне непрерывное сечение многозначного отобраэсения $F$. Если существует вполне непрерывное сечение $\tilde{f}: U \rightarrow E$ многозначного отображения $\widetilde{F}$, совпадающее $c f$ на мнохестве $X$, то отобрахсение $f$ имеет неподвижную точку.

ДокАЗАтЕльство. Пусть $V \subset E$-открытоемножество такое, что $X \subset V \subset \bar{V} \subset U$. Рассмотрим вполне непрерьвное отображение $f_{0}: \bar{V} \rightarrow E, f_{0}(x)=x_{0}$. Тогда вращение $\gamma\left(\varphi_{0}, \partial V\right)$ векторного поля $\varphi_{0}=i-f_{0}$ равно 1 (см., например, [12]). Рассмотрим отображение

$$
\Phi(\lambda, x)=x-\lambda f_{0}(x)-(1-\lambda) \tilde{f}(x), \quad \Phi:[0,1] \times \bar{V} \rightarrow E .
$$

Так как $\lambda f_{0}(x)+(1-\lambda) f(x) \in \widetilde{F}(x)$ для любого $\lambda \in[0,1]$, то $\Phi(\lambda, x) \neq 0$ для любого $x \in \partial V$. Следовательно, $\Phi$ является гомотопией, соединяющей поля $\varphi_{0}$ и $\varphi=i-\tilde{f}$. Так как вращение вполне непрерьвных векторных полей инвариантно при гомотопиях, то $\gamma(\varphi, \partial V)=1$. Тогда отображение $\tilde{f}$ обязано иметь в множестве $V$ неподвижную точку. Так как $\tilde{f}$ является сечением $\widetilde{F}$, то неподвижная точка должна принадлежать множеству $X$. В силу того, что $\tilde{f}$ совпадает с $f$ на $X$, получаем, что неподвижную точку имеет отображение $f$. Лемма доказана.

Пусть $E$ - банахово пространство, $X$ - вьпуклое множество в $E$. Обозначим через

$$
S_{X}(x):=\bigcup_{h>0} \frac{1}{h}(X-x)
$$

конус, порожденньй $X-x$, и через

$$
T_{X}(x):=\overline{\bigcup_{h>0} \frac{1}{h}(X-x)}
$$

обозначим его замыкание. Множество $T_{X}(x)$ назьвается касательнымм конусом к $X$ в точке $x$.

Таким образом, для любой точки $v \in S_{X}(x)$ существует $h>0$ такое, что $x+h v \in X$. Нетрудно доказать, что множества $S_{X}(x)$ и $T_{X}(x)$ являются выпуклыми.

Рассмотрим многозначное отображение $T: X \rightarrow \operatorname{Cv}(E), T(x)=T_{X}(x)$.

ЛЕмма 2. Многозначное отображсение Т является полунепрерывным снизу. 
ДокАЗАТЕЛЬСтво. Пусть $V$ - произвольное открытое множество в $E$. Рассмотрим

$$
T_{\Pi}^{-1}(V)=\{x \in X \mid T(x) \cap V \neq \varnothing\}
$$

и покажем, что это множество является открытым. Пусть точка $x_{0} \in T_{\Pi}^{-1}(V)$, тогда существует точка $y_{0} \in(T(x) \cap V)$. Рассмотрим $\varepsilon>0$ такое, что $U_{2 \varepsilon}\left(y_{0}\right) \subset V$. В силу определений множеств $S_{X}\left(x_{0}\right)$ и $T_{X}\left(x_{0}\right)$ существует точка $z_{0} \in\left(S_{X}\left(x_{0}\right) \cap U_{\varepsilon}\left(y_{0}\right)\right)$. Следовательно, существуют точка $u \in X$ и число $h>0$ такие, что $z_{0}=\left(u-x_{0}\right) / h$. Пусть $x_{1} \in X$, рассмотрим точку

$$
z_{1}=\frac{1}{h}\left(u-x_{1}\right) \in S_{X}\left(x_{1}\right) .
$$

Тогда

$$
\left\|z_{0}-z_{1}\right\|=\frac{1}{h}\left\|x_{1}-x_{0}\right\| .
$$

Если $\left\|x_{0}-x_{1}\right\|<\delta$, где $0<\delta<h \varepsilon$, то $S_{X}\left(x_{1}\right) \cap U_{\varepsilon}\left(y_{0}\right) \neq \varnothing$. Так как

$$
\left(S_{X}\left(x_{1}\right) \cap U_{\varepsilon}\left(y_{0}\right)\right) \subset\left(T_{X}\left(x_{1}\right) \cap U_{2 \varepsilon}\left(y_{0}\right)\right) \subset\left(T\left(x_{1}\right) \cap V\right),
$$

то множество $T_{\Pi}^{-1}(V)$ содержит окрестность $U_{\delta}\left(x_{0}\right) \cap X$, что и доказывает открытость множества $T_{\Pi}^{-1}(V)$. Лемма доказана.

Заметим, что другими, более сложными, методами эта лемма была доказана в [4].

Пусть $X$ - выпуклое подмножество гильбертова пространства $H$. Рассмотрим отображение $r: H \rightarrow X$, сопоставляющее каждой точке $x \in H$ ближайший к ней элемент из $X$, т.е.

$$
\|x-r(x)\|=\min _{y \in X}\|x-y\| .
$$

Хорошо известно, что такое отображение существует и непрерьвно. Такоеотображение назьвается метрической проекиией.

ЛЕмма 3. Для любой точки $x \in H$ ближайшим $\kappa$ ней әлементом из множества $P(r(x))=r(x)+T_{X}(r(x))$ является точка $r(x)$, т.е.

$$
\|x-r(x)\|=\min _{w \in P(r(x))}\|x-w\|
$$

ДокАЗАТЕЛЬСТво. Известно, что точка $v \in T_{X}(x)$ в том и только том случае, если $(y-x, v) \leqslant 0$ для любого $y \in r^{-1}(x)$ (см., например, [1]).

Пусть $w \in P(r(x))$, тогда $w=r(x)+v$, где $v \in T_{X}(r(x))$. Оценим,

$$
\|x-w\|^{2}=(x-r(x)-v, x-r(x)-v)=\|x-r(x)\|^{2}+\|v\|^{2}-2(x-r(x), v) .
$$

Очевидно, что минимум левой части достигается при $v=0$, что и доказывает лемму.

Следствием этой леммы является следующее утверждение.

ЛЕмма 4. Если точка $x \in U_{\varepsilon}(P(r(x)))$, то $x \in U_{\varepsilon}(X)$. 
Теорема 5. Пусть $X$ - выпуклое замкнутое ограниченное подмножсество гильбертова пространства $H$. Пусть $F: X \rightarrow \mathrm{Kv}(H)$ - вполне непрерывное многозначное отображсние такое, что для любой точки $x \in X$ пересечение $F(x) \cap$ $\left(x+T_{X}(x)\right) \neq \varnothing$. Тогда $F$ имеет неподвихную точку.

ДокАЗАТЕЛЬСтво. Первый этап. Пусть $\varepsilon$ - произвольное положительное число. Рассмотрим полунепрерывное снизу многозначное отображение $F_{\varepsilon}: X \rightarrow \mathrm{Cv}(H)$, удовлетворяющее условиям теоремы 2 , т.е. $F(x) \subset F_{\varepsilon}(x)$ для любого $x \in X$; график $\Gamma_{X}\left(F_{\varepsilon}\right) \subset U_{\varepsilon}\left(\Gamma_{X}(F)\right) ; F_{\varepsilon}(X) \subset \overline{\mathrm{co}}(F(X))$.

Рассмотрим также многозначное отображение $P: X \rightarrow \mathrm{Cv}(H)$, определенное условием, $P(x)=x+T_{X}(x)$. В силу леммы 2 отображение $P$ является полунепрерывныпм снизу. Очевидно, что для любого $x \in X$ пересечение $F_{\varepsilon}(X) \cap P(x) \neq \varnothing$. Тогда в силу теоремы 4 существует однозначное непрерывное отображение $f_{\varepsilon}: X \rightarrow H$ такое, что:

(i) $f_{\varepsilon}(x) \in F_{\varepsilon}(x)$ для любого $x \in X$;

(ii) $f_{\varepsilon}(x) \in U_{\varepsilon}(P(x))$ для любого $x \in X$;

(iii) отображение $f_{\varepsilon}$ является вполне непрерьвньм.

Справедливость условия (iii) вытекает из того, что

$$
f_{\varepsilon}(X) \subset F_{\varepsilon}(X) \subset \overline{c o}(F(X)) .
$$

Пусть $r: H \rightarrow X$ - метрическая проекция. Рассмотрим непрерывное однозначное отображение $\tilde{f}_{\varepsilon}: H \rightarrow H$, определенное условием $\tilde{f}_{\varepsilon}(x)=f_{\varepsilon}(r(x))$. Это отображение является вполне непрерывным и удовлетворяет условию

$$
\tilde{f}_{\varepsilon}(x) \in U_{\varepsilon}(P(r(x))) .
$$

Рассмотрим многозначное отображение $P_{\varepsilon}: H \rightarrow V(H), P_{\varepsilon}(x)=U_{\varepsilon}(P(r(x)))$. Нетрудно проверить, что многозначное отображение $P_{\varepsilon}$ удовлетворяет условиям леммы 1 на множестве $\overline{U_{\varepsilon}(X)}$. Действительно, в силу леммы 4 отображение $P_{\varepsilon}$ не имеет неподвижных точек вне $U_{\varepsilon}(X)$. В качестве точки $x_{0}$ можно взять любую точку множества $X$, звездность образов этого отображения вытекает из вьпуклости множеств $\Phi_{\varepsilon}(x)$. Следовательно, отображение $\tilde{f}_{\varepsilon}$ имеет неподвижную точку $x_{\varepsilon}$ и $x_{\varepsilon} \in U_{\varepsilon}(X)$.

Обозначим точку $r\left(x_{\varepsilon}\right)=y_{0}$, тогда эта точка удовлетворяет следующим условиям, $\left\|x_{\varepsilon}-y_{0}\right\|<\varepsilon$ и $f_{\varepsilon}\left(y_{0}\right)=x_{\varepsilon}$. Следовательно, $\left\|f_{\varepsilon}\left(y_{0}\right)-y_{0}\right\|<\varepsilon$.

$\mathrm{C}$ другой стороны, так как

$$
\Gamma_{X}\left(f_{\varepsilon}\right) \subset \Gamma_{X}\left(F_{\varepsilon}\right) \subset U_{\varepsilon}\left(\Gamma_{X}(F)\right),
$$

то существуют точки $x_{0} \in X$ и $z_{0} \in F\left(x_{0}\right)$ такие, что $\left\|y_{0}-x_{0}\right\|<\varepsilon$ и $\left\|f_{\varepsilon}\left(y_{0}\right)-z_{0}\right\|<\varepsilon$. Тогда

$$
\begin{aligned}
\rho\left(x_{0}, F\left(x_{0}\right)\right) & =\min _{y \in F\left(x_{0}\right)}\left\|x_{0}-y\right\| \leqslant\left\|x_{0}-z_{0}\right\| \\
& \leqslant\left\|x_{0}-y_{0}\right\|+\left\|y_{0}-f_{\varepsilon}\left(y_{0}\right)\right\|+\left\|f_{\varepsilon}\left(y_{0}\right)-z_{0}\right\|<3 \varepsilon .
\end{aligned}
$$

Таким образом, доказано, что для любого $\varepsilon>0$ существует точка $x_{0} \in X$ такая, что $\rho\left(x_{0}, F\left(x_{0}\right)\right)<3 \varepsilon$. 
Второй этап. Рассмотрим последовательность положительных чисел $\left\{\varepsilon_{n}\right\}_{n=1}^{\infty}$, стремящуюся к 0 . Каждому числу $\varepsilon_{n}$ отвечает точка $x_{n} \in X$ такая, что $\rho\left(x_{n}, F\left(x_{n}\right)\right)<3 \varepsilon_{n}$. Рассмотрим последовательность $\left\{y_{n}\right\}_{n=1}^{\infty}, y_{n} \in F\left(x_{n}\right)$ и $\left\|x_{n}-y_{n}\right\|<3 \varepsilon_{n}$. Тогда последовательность $\left\{y_{n}\right\}_{n=1}^{\infty} \subset \overline{F(X)}$. В силу того, что многозначное отображение $F$ является вполне непрерывным, без ограничения общности можно считать, что последовательность $\left\{y_{n}\right\}_{n=1}^{\infty}$ сходится к некоторой точке $y_{*}$. Следовательно, последовательность $\left\{x_{n}\right\}_{n=1}^{\infty}$ также сходится к $y_{*}$. Так как множество $X$ является замкнутым, то $y_{*} \in X$. Тогда из замкнутости графика многозначного отображения $F$ вытекает, что $y_{*} \in F\left(y_{*}\right)$. Теорема доказана.

ЗАмЕчАнИЕ. Остается открытым вопрос о справедливости теоремы 5 для произвольного банахова пространства.

\section{СПИСОК ЦИТИРОВАННОЙ ЛИТЕРАТУРЫ}

[1] Обэн ЖК.-П., Экланд И. Прикладной нелинейный анализ. М.: Мир, 1988.

[2] Ben-El-Mechaiekh H., Kryszewski W. Equilibria of set-valued maps on nonconvex domains // Trans. Amer. Math. Soc. 1997. V. 349. № 10. P. 4159-4179.

[3] Aubin J.-P., Cellina A. Differential Inclusions. Set-Valued Maps and Viability Theory. Grundlehren Math. Wiss. V. 264. Berlin: Springer-Verlag, 1984.

[4] Aubin J.-P., Frankowska H. Set-Valued Analysis. Boston: Birkhäuser, 1990.

[5] Обэн ЖК.-П. Нелинейньй анализ и его экономические приложения. М.: Мир, 1988.

[6] Борисович Ю. Г., Гельман Б. Д., Мышкис А. Д., Обуховский В.В. Топологические методы в теории неподвижных точек многозначных отображений // УМН. 1980. Т. 35. № 1. C. 59-126.

[7] Борисович Ю.Г., Гельман Б. Д., Мышкис А. Д., Обуховский В.В. Многозначные отображения // Итоги науки и техн. Матем. анализ. Т. 19. М.: ВИНИТИ, 1982. C. 127-229.

[8] Реповш Д., Семенов П. В. Теория Э. Майкла непрерьвных селекций. Развитие и приложения // УМН. 1994. Т. 49. №6. С. 151-188.

[9] Repovs D., Semenov P. V. Continuous Selections of Multivalued Mappings. Mathematics and its Applications. V. 455. Dordrecht: Kluwer, 1998.

[10] Борисович Ю. Г., Гельман Б. Д., Мышкис А. Д., Обуховский В. В. Введение в теорию многозначных отображений. Воронеж: Изд-во ВГУ, 1986.

[11] Michael E. Continuous selections, 1 // Ann. of Math. 1956. V. 63. № 2. P. 361-382.

[12] Красносельский М. А., Забрейко П. П. Геометрические методы нелинейного анализа. М.: Наука, 1975. 